\title{
Temperature-driven nonclassical light
}

\author{
F. Pennini, ${ }^{1,2}$ A. Plastino, ${ }^{1,3}$ and G. L Ferri ${ }^{4}$ \\ ${ }^{1}$ Instituto de Física, CCT La Plata, CONICET, Universidad Nacional de La Plata, C.C. 727, 1900, La Plata, Argentina \\ ${ }^{2}$ Departamento de Física, Universidad Católica del Norte, Av. Angamos 0610, Antofagasta, Chile \\ ${ }^{3}$ Physics Department and IFISC-CSIC, University of Balearic Islands, 07122, Palma de Mallorca, Spain \\ ${ }^{4}$ Facultad de Ciencias Exactas y Naturales, Universidad Nacional de La Pampa, Peru y Uruguay, 6300, Santa Rosa, La Pampa, Argentina
}

(Received 7 December 2012; revised manuscript received 21 March 2013; published 21 June 2013)

\begin{abstract}
With reference to two well known scenarios, we discuss, for nonclassical light, the competition between quantum and thermal effects. It is seen that for nonclassical light to be produced some amount of temperatureinduced disorder is needed plus quantum fluctuations of order $\hbar$ squared.
\end{abstract}

DOI: 10.1103/PhysRevE.87.064101

PACS number(s): 05.30.-d, 03.65.Sq, 03.67.-a

\section{INTRODUCTION}

Nonclassical light is a subject of much current interest, with many fascinating applications (see Ref. [1], and references therein). Thus, several branches of quantum optics, from nonlinear optics to laser physics and cavity quantum optics, are actively engaged in a variety of processes producing nonclassical light. Such radiation fields attract attention because they provide a platform for testing fundamental concepts of quantum theory and also for applications of importance, such as precision measurements in interferometry [2]. Moreover, rapidly developing areas of quantum computation and information theory have kindled further interest in generating and manipulating nonclassical radiation fields (we speak of quantum, continuous variable states). These states are promising candidates for many applications of quantum information technology [3-5].

Generally, a nonclassical state is recognized as one which cannot be written as a statistical mixture of coherent states. It is accepted that the nonexistence of a well defined GlauberSudarshan $P$ function [6] renders a given state nonclassical. However, this identification poses operational difficulties, as it requires complete information regarding the state to be examined (so that its $P$ function can be reconstructed). Several operational criteria, equivalent to the one based on the $P$ function, can be used to distinguish between classical and nonclassical states in experimental measurements and have been proposed from the early days of quantum optics. Such signatures of nonclassicality, verifiable in simple empirical settings are, for instance, antibunching [7] and sub-Poissonian photon statistics [8], squeezing [9], photon number oscillations $[10,11]$, etc.

The aim of the present communication is to enter the above nonclassicality discussion by considering, as a main tool, the Mandel parameter. Two scenarios are envisaged:

(i) the Jaynes-Cummings model, and

(ii) another in which disorder, as generated by temperature, competes with order, generated by molecular alignment. This opposition is provided an optical Kerr medium.

We start our presentation with a Mandel recapitulation in Sec. II. After some preliminary considerations in Sec. III, we present our Brief Report's contributions in Secs. IV, V, and VI. In the first case, via the treatment of the Mandel parameter for a Kerr medium, proceeding with the orthodox, quantum-statistical canonical description. Next, we address in Secs. V and VI the Jaynes-Cummings (JC) model without and with (VI) a Kerr-like term, so as to ascertain whether nonclassical light emerges in these two scenarios as well. Finally, we conclude in Sec. VII.

\section{MANDEL PARAMETER}

Our main protagonist is a convenient noise indicator of a nonclassical field, the so-called Mandel parameter, introduced by Mandel in Ref. [12]. It reads [13]

$$
\mathcal{Q}=\frac{(\Delta \hat{n})^{2}}{\langle\hat{n}\rangle}-1 \equiv \mathcal{F}-1,
$$

closely related to the normalized variance (also called the quantum Fano factor $\mathcal{F}$ [14]) $\mathcal{F}=(\Delta \hat{n})^{2} /\langle\hat{n}\rangle$ of the photon distribution.

(i) For $\mathcal{F}<1(\mathcal{Q} \leqslant 0)$, emitted light is referred to as subPoissonian since it has photo-count noise smaller than

(ii) that of coherent (ideal laser) light with the same intensity $(\mathcal{F}=1 ; \mathcal{Q}=0)$, whereas

(iii) for $\mathcal{F}>1,(\mathcal{Q}>0)$ the light is called super-Poissonian, exhibiting photo-count noise higher than the coherent-light noise.

Of course, one wishes to minimize the Fano factor. If for the photon number operator $\hat{n}$ the fluctuations in $\hat{n}$ vanish, the Mandel parameter becomes $\mathcal{Q}=-1(\mathcal{F}=0)$.

\section{KERR EFFECT}

Our main physical ingredient in this presentation is the Kerr effect, also called the quadratic electro-optic effect, discovered in 1875 by the Scottish physicist John Kerr. It refers to a change in the refractive index of a material in response to an applied electric field. Many materials exhibit a Kerr effect, but certain liquids display it more strongly than others. For an appropriate medium the difference in index of refraction, $\Delta n$, is given by

$$
\Delta n=\lambda K \mathcal{E}^{2},
$$

where $\lambda$ is the wavelength of the light, $K$ the so-called Kerr constant, and $\mathcal{E}$ is the strength of the electric field. The optical Kerr effect manifests itself temporally as a self-phase modulation, a self-induced phase and frequency shift of a pulse of light as it travels through a medium. Such process, together with dispersion, can produce optical solitons. In materials with high Kerr constants the molecules will tend to align with 
the applied electric field [16]. Thus, "order" can be said to augment.

The Hamiltonian for this system is

$$
\begin{aligned}
\hat{H}_{\chi} & =\hbar \omega\left(\hat{a}^{\dagger} \hat{a}+\frac{1}{2}\right)+\hbar \chi\left(\hat{a}^{\dagger}\right)^{2}(\hat{a})^{2} \\
& =\hbar \omega(\hat{n}+1 / 2)+\hbar \chi\left(\hat{n}^{2}-\hat{n}\right),
\end{aligned}
$$

where the number operator is $\hat{n}=\hat{a}^{\dagger} \hat{a}$, and the nonlinear coupling coefficient $\chi$ (a positive real quantity that characterizes the Kerr environment) is related to the Kerr medium third-order susceptibility [15-17].

In a beautiful study [15], it is shown that Kerr states exhibit Poissonian photon statistics and their Mandel parameter is identically zero. In order to modify this environment, Ref. [15] appeals to the concept of displaced Kerr states to produce sub-Poisson and super-Poisson statistics, depending on certain parameters that characterizing the displaced Kerr states. We readdress the question here by recourse to two alternatives, with a view to discuss also the order-disorder competition generated by the opposition: temperature vs Kerr interaction.

\section{QUANTUM STATISTICAL TREATMENT}

The semiclassical approach allows one to see quantum effects of order $\hbar$ only (see, for instance, Refs. [18,19], and references therein). These $\hbar$ effects are, however, not strong enough to yield negative values for the Mandel factor. Consequently, one needs to add $\hbar^{2}$ (and higher order) effects to the theoretical discussion, which can straightforwardly be accomplished by any purely quantal treatment. Accordingly, we choose to work with the canonical strictures of quantum statistical mechanics.
Let $|n\rangle$ be the eigenvector of the Hamiltonian (3), whose energy spectrum is $\varepsilon_{n}$, defined as

$$
\varepsilon_{n}=\hbar \omega(n+1 / 2)+\hbar \chi\left(n^{2}-n\right)
$$

We compute expectation values as follows:

$$
\left\langle\hat{n}^{2}\right\rangle=Z^{-1} \operatorname{Tr}\left(e^{-\beta \hat{H}_{x}} \hat{n}^{2}\right)=Z^{-1} \sum_{n=0}^{\infty} n^{2} e^{-\beta \varepsilon_{n}},
$$

and

$$
\langle\hat{n}\rangle=Z^{-1} \operatorname{Tr}\left(e^{-\beta \hat{H}_{\chi}} \hat{n}\right)=Z^{-1} \sum_{n=0}^{\infty} n e^{-\beta \varepsilon_{n}},
$$

where

$$
Z=\operatorname{Tr}\left(e^{-\beta \hat{H}_{\chi}}\right)=\sum_{n=0}^{\infty} e^{-\beta \varepsilon_{n}}
$$

is the partition function.

Introducing now Eqs. (5) and (6) into Eq. (1), after some manipulations we finally find for the Mandel parameters the expression

$$
\mathcal{Q}=\frac{\sum_{n=0}^{\infty} n^{2} \rho_{n}-\left(\sum_{n=0}^{\infty} n \rho_{n}\right)^{2}}{\sum_{n=0}^{\infty} n \rho_{n}}-1,
$$

where we have defined the probabilities $\rho_{n}$ as

$$
\rho_{n}=\frac{e^{-\beta \hbar \omega n-\beta \hbar \chi\left(n^{2}-n\right)}}{\sum_{n=0}^{\infty} e^{-\beta \hbar \omega n-\beta \hbar \chi\left(n^{2}-n\right)}} .
$$

Notice that if $\chi$ tends to zero we recover the Mandel parameter for the harmonic oscillator $(\mathrm{HO})$. In fact, we have $\rho_{n}^{(\mathrm{HO})}=$ $\exp (-\beta \hbar \omega n) \sum_{n=0}^{\infty} \exp (-\beta \hbar \omega n)$ so that

$$
\mathcal{Q}^{(\chi \rightarrow 0)}=\frac{\sum_{n=0}^{\infty} n^{2} e^{-\beta \hbar \omega n}-\left(\sum_{n=0}^{\infty} e^{-\beta \hbar \omega n}\right)^{-1}\left(\sum_{n=0}^{\infty} n e^{-\beta \hbar \omega n}\right)^{2}}{\sum_{n=0}^{\infty} n e^{-\beta \hbar \omega n}}-1,
$$

and performing the pertinent sums we arrive at

$$
\mathcal{Q}^{(\chi \rightarrow 0)}=\frac{e^{-\beta \hbar \omega}}{1-e^{-\beta \hbar \omega}},
$$

which is the well known $\mathrm{HO}$ expression for Mandel parameters.

In our graphs we will employ the dimensionless temperature $t=k_{B} T / \hbar \omega$, with $k_{B}$ being Boltzmann's constant.

As illustrated in Fig. 1, we achieve our sub-Poissonian goal. There exists a range of $t$ and $\chi$ values for which $\mathcal{Q}$ negativity ensues. Contour curves in the $t-\chi$ plane are presented in Fig. 2, and it becomes clear that finite $t$ are needed for negative Mandel factors to ensue.

Figure 3 plots the Mandel factor vs $t$ for several Kerr strengths, while Fig. 4 is a zoom of the bottom left corner of Fig. 3. These graphs permit a better visualization of the findings of Fig. 2. Notice the curious fact that nonclassical light emerges after some finite values for both $t$ and the Kerr strength are reached, that is, threshold values for both $t$ and $\chi$ are detected.

\section{JAYNES-CUMMINGS MODEL}

Cavity quantum electrodynamics (cavity QED) deals with the interaction of atoms with a single electromagnetic field mode. Original references are, for instance, [20,21]. A nice review is that of Ref. [22]. The JC model is a two-level system (consisting of states $|g\rangle,|e\rangle$ separated by an energy gap $\hbar \omega_{A}$ ) and interacting with a single electromagnetic field mode. The pertinent Hamiltonian reads [22]

$$
\hat{H}_{\mathrm{JC}}=\hat{H}_{\text {field }}+\hat{H}_{\text {atom }}+\hat{H}_{\text {int }},
$$

where

$$
\hat{H}_{\text {field }}=\hbar \omega \hat{a}^{\dagger} \hat{a}
$$

describes a longitudinal quasimode in the cavity and

$$
\hat{H}_{\text {atom }}=\hbar \omega_{A} \hat{\sigma}_{e g} \hat{\sigma}_{g e},
$$

refers to the noninteracting two-level atom. The spin-flip operators are defined as $\hat{\sigma}_{g e}=|g\rangle\langle e|$. The operators $\hat{a}, \hat{a}^{\dagger}$ are given by the decomposition of our single-mode field 


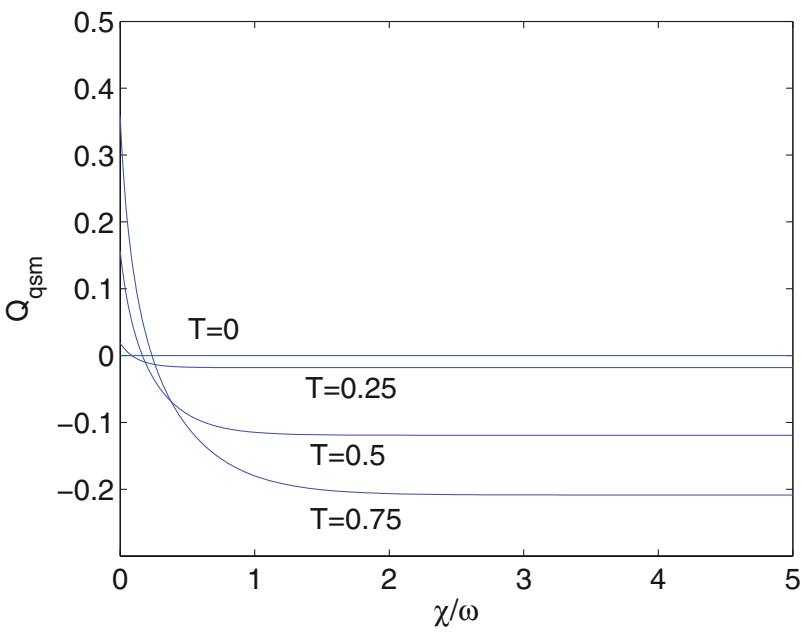

FIG. 1. (Color online) $\mathcal{Q}$ as a function of $\chi / \omega$ for several values of temperature, i.e., $t=0,0.25,0.5,0.75$.

(one chooses the $x$ axis in such a way that there is a standing-wave node at $x=0$. Accordingly, a sine appears in the decomposition)

$$
\hat{E}=\varepsilon \sin (k x)\left(\hat{a}+\hat{a}^{\dagger}\right), \quad \varepsilon=\sqrt{\frac{\hbar \omega}{2 \epsilon_{0} V}},
$$

$V$ being the cavity volume and $\epsilon_{0}$ the electric constant.

Using Eq. (15) one obtains for the interaction energy in the dipole approximation the expression

$$
\begin{aligned}
\hat{H}_{\mathrm{int}} & =-\gamma \varepsilon \sin (k x)\left(\hat{\sigma}_{e g}+\hat{\sigma}_{g e}\right)\left(\hat{a}+\hat{a}^{\dagger}\right) \\
& \equiv g \hbar\left(\hat{a} \hat{\sigma}_{e g}+\hat{a}^{\dagger} \hat{\sigma}_{g e}\right)
\end{aligned}
$$

where in the last step the rotating wave approximation (RWA) is appealed to. The coupling constant $g$ is given by

$$
g=-\frac{\varepsilon \gamma}{\hbar} \sin (k x), \quad \gamma=\langle e|e \widehat{\vec{r}}| g\rangle .
$$

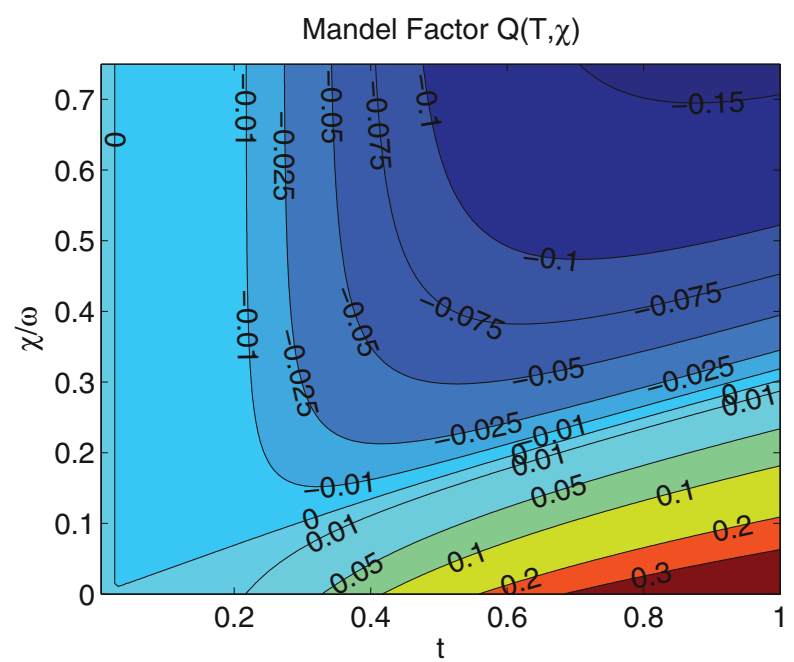

FIG. 2. (Color online) Mandel parameter $\mathcal{Q}$ in the plane $\chi / \omega-t$. Note that for significantly negative $\mathcal{Q}$ values, like, for instance, -0.06 , one definitely requires a finite temperature.

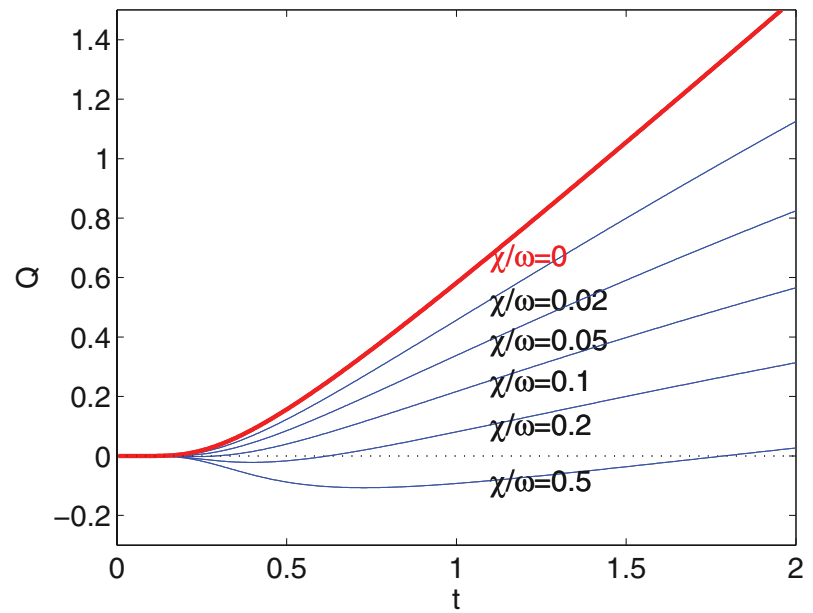

FIG. 3. (Color online) $\mathcal{Q}$ as a function of $t$ for several values of $\chi / \omega$, i.e., $\chi / \omega=0.02,0.05,0.1,0.2,0.5$. Negative Mandel factors demand finite temperatures.

The Hamiltonian (12) in the RWA is the Jaynes-Cummings one. It is analytically solvable, which can be easily seen when restricting ourselves on the invariant subspace $\mathcal{N}=$ $\{|n+1\rangle|g\rangle,|n\rangle|e\rangle\}: \mathcal{H} \mathcal{N} \subset \mathcal{N}$, i.e., $\mathcal{H}$ is block diagonal. Exact diagonalization of the $2 \times 2$ blocks yields the eigenstates (dressed states)

$$
\begin{aligned}
& |+, n\rangle=\sin \left(\theta_{n}\right)|n\rangle|e\rangle+\cos \left(\theta_{n}\right)|n+1\rangle|g\rangle \\
& |-, n\rangle=\cos \left(\theta_{n}\right)|n\rangle|e\rangle-\sin \left(\theta_{n}\right)|n+1\rangle|g\rangle,
\end{aligned}
$$

with

$$
\begin{aligned}
\cos \left(\theta_{n}\right) & =\sqrt{\frac{\Omega_{n}-\delta}{2 \Omega_{n}}}, \quad \sin \left(\theta_{n}\right)=\sqrt{\frac{\Omega_{n}+\delta}{2 \Omega_{n}}} \\
\Omega_{n} & =\sqrt{4 g^{2}(n+1)+\delta^{2}}, \delta=\omega_{A}-\omega .
\end{aligned}
$$

The eigenenergies of these dressed states are

$$
E_{ \pm, n}=\frac{1}{2} \hbar \omega_{A}+(n+1) \hbar \omega \pm \frac{1}{2} \hbar \Omega_{n}
$$

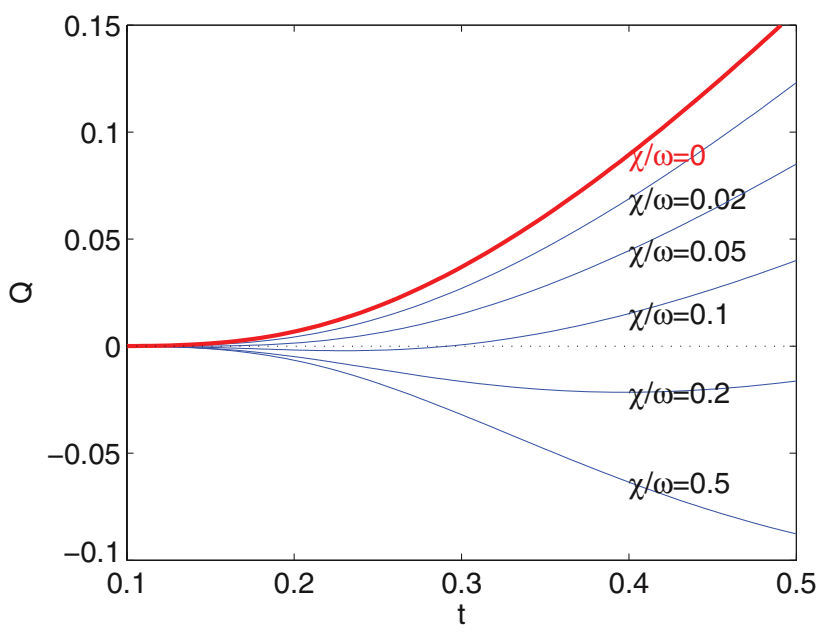

FIG. 4. (Color online) Represents a zoom of $\mathcal{Q}$ as a function of $t$ of the bottom left corner of Fig. 3. 
For simplicity, in our calculations we will consider the resonant case in which $\delta=0$. This implies $\omega_{A}=\omega$. In this situation we have

$$
\Omega_{n}=2 g \sqrt{n+1},
$$

the energy spectrum being

$$
E_{ \pm, n}=\left(n+\frac{3}{2}\right) \hbar \omega \pm \hbar g \sqrt{n+1}
$$

We can express expectation values as follows:

$$
\begin{aligned}
\left\langle\hat{n}^{2}\right\rangle & =Z^{-1} \operatorname{Tr}\left(e^{-\beta \hat{H}_{\mathrm{JC}}} \hat{n}^{2}\right)=Z^{-1} \sum_{n=0}^{\infty} n^{2}\left(e^{-\beta E_{+, n}}+e^{-\beta E_{-, n}}\right) \\
& \equiv 2 e^{-3 \beta \hbar \omega / 2} Z^{-1} \sum_{n=0}^{\infty} n^{2} e^{-\beta \hbar \omega n} \cosh (\beta \hbar g \sqrt{n+1})
\end{aligned}
$$

and

$$
\begin{aligned}
\langle\hat{n}\rangle & =Z^{-1} \operatorname{Tr}\left(e^{-\beta \hat{H}_{\mathrm{JC}}} \hat{n}\right)=Z^{-1} \sum_{n=0}^{\infty} n\left(e^{-\beta E_{+, n}}+e^{-\beta E_{-, n}}\right) \\
& \equiv 2 e^{-3 \beta \hbar \omega / 2} Z^{-1} \sum_{n=0}^{\infty} n e^{-\beta \hbar \omega n} \cosh (\beta \hbar g \sqrt{n+1}),
\end{aligned}
$$

where the partition function is

$$
\begin{aligned}
Z & =\operatorname{Tr}\left(e^{-\beta \hat{H}_{\mathrm{JC}}}\right)=\sum_{n=0}^{\infty}\left(e^{-\beta E_{+, n}}+e^{-\beta E_{-, n}}\right) \\
& \equiv 2 e^{-3 \beta \hbar \omega / 2} \sum_{n=0}^{\infty} e^{-\beta \hbar \omega n} \cosh (\beta \hbar g \sqrt{n+1})
\end{aligned}
$$

Reordering things, and after a bit of algebra, we can rewrite these mean values in the fashion

$$
\begin{aligned}
\left\langle\hat{n}^{2}\right\rangle & =\sum_{n=0}^{\infty} n^{2} \rho_{n}^{*}, \\
\langle\hat{n}\rangle & =\sum_{n=0}^{\infty} n \rho_{n}^{*},
\end{aligned}
$$

where in this case

$$
\rho_{n}^{*}=\frac{e^{-\beta \hbar \omega n} \cosh (\beta \hbar g \sqrt{n+1})}{\sum_{n=0}^{\infty} e^{-\beta \hbar \omega n} \cosh (\beta \hbar g \sqrt{n+1})},
$$

which is to be compared to the probabilities (9)

$$
\rho_{n}=\frac{e^{-\beta \hbar \omega n-\beta \hbar \chi\left(n^{2}-n\right)}}{\sum_{n=0}^{\infty} e^{-\beta \hbar \omega n-\beta \hbar \chi\left(n^{2}-n\right)}},
$$

in order to appreciate the different manners in which the Kerr interaction and the cavity one modify the harmonic oscillator probabilities. The latter is rather a "hyperbolic" modulation while the first behaves as an actual interaction added to that of the HO. This is because if we deal with a decomposable Hamiltonian $H=H_{A}+H_{B}$, we expect the canonical probability distribution to be, up to a normalization factor, of the form

$$
\rho \sim \exp (-\beta H)=\exp \left[-\beta\left(H_{A}+H_{B}\right)\right],
$$

and only (9) is of such a form.

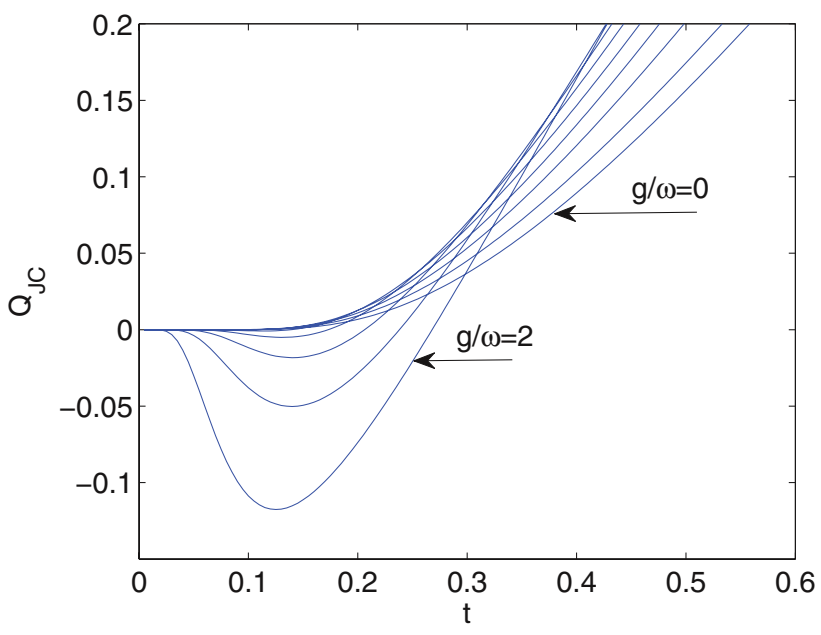

FIG. 5. (Color online) $\mathcal{Q}_{\mathrm{JC}}$ as a function of $t$ for several values of $g / \omega$, i.e., $g / \omega=0$ to 2 .

Finally, introducing Eqs. (27) and (28) into the definition (8) and replacing $\rho_{n}^{*}$ by $\rho_{n}$, we arrive at the Mandel parameter's expression for the Jaynes-Cummings model. One has

$$
\mathcal{Q}_{\mathrm{JC}}=\frac{\sum_{n=0}^{\infty} n^{2} \rho_{n}^{*}-\left(\sum_{n=0}^{\infty} n \rho_{n}^{*}\right)^{2}}{\sum_{n=0}^{\infty} n \rho_{n}^{*}}-1
$$

In Fig. 5 we depict the Mandel parameter for the JaynesCummings model in terms of dimensionless temperature $t$ for several values of the coupling constant $g$. The case $g / \omega=0$ corresponds to the harmonic oscillator model. In these curves we clearly see that, at some finite temperatures, the Mandel parameter is negative for appropriate $g$ values and thus nonclassical light ensues.

\section{JAYNES-CUMMINGS MODEL PLUS KERR-LIKE TERM}

Let us now add to the JC Hamiltonian a Kerr-like term, so that a new Hamiltonian ensues

$$
\hat{H}=\hat{H}_{\mathrm{JC}}+\hbar \chi \hat{n}^{2},
$$

with eigenenergies

$$
E_{ \pm, n}^{*}=\left(n+\frac{3}{2}\right) \hbar \omega \pm \hbar g \sqrt{n+1}+\hbar \chi n^{2} .
$$

The relevant expectation values are found in similar way as previous section

$$
\left\langle\hat{n}^{2}\right\rangle=2 e^{-3 \beta \hbar \omega / 2} Z^{-1} \sum_{n=0}^{\infty} n^{2} e^{-\beta \hbar \omega n-\beta \hbar \chi n^{2}} \cosh (\beta \hbar g \sqrt{n+1}),
$$

and

$$
\langle\hat{n}\rangle=2 e^{-3 \beta \hbar \omega / 2} Z^{-1} \sum_{n=0}^{\infty} n e^{-\beta \hbar \omega n-\beta \hbar \times n^{2}} \cosh (\beta \hbar g \sqrt{n+1}),
$$




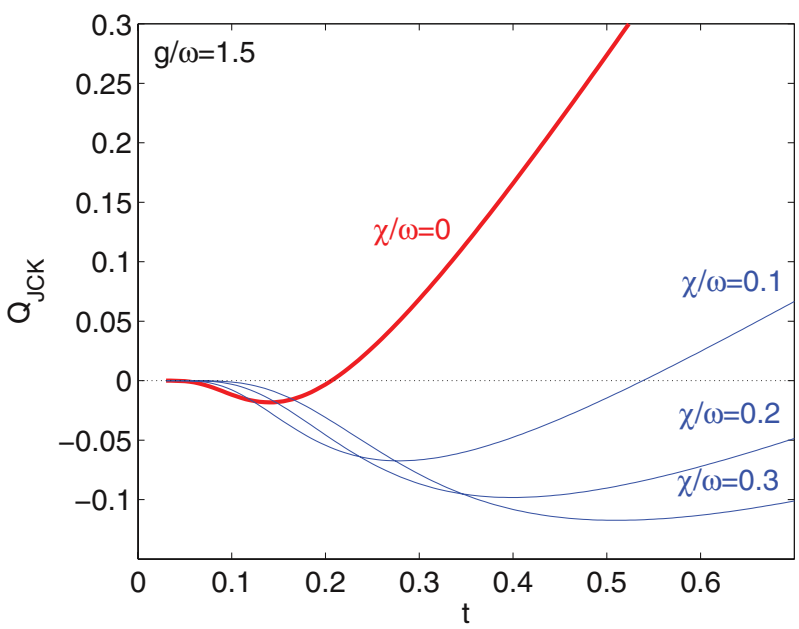

FIG. 6. (Color online) $\mathcal{Q}_{\mathcal{J C K}}$ as a function of $t$ for $g / \omega=1.5$. $\chi / \omega$ ranges from 0.1 to 0.3 .

with a partition function

$$
Z=2 e^{-3 \beta \hbar \omega / 2} \sum_{n=0}^{\infty} e^{-\beta \hbar \omega n-\beta \hbar \times n^{2}} \cosh (\beta \hbar g \sqrt{n+1})
$$

Also, these mean values are of the form

with

$$
\begin{aligned}
\left\langle\hat{n}^{2}\right\rangle & =\sum_{n=0}^{\infty} n^{2} \rho_{n}^{* *}, \\
\langle\hat{n}\rangle & =\sum_{n=0}^{\infty} n \rho_{n}^{* *},
\end{aligned}
$$

$$
\rho_{n}^{* *}=\frac{e^{-\beta \hbar \omega n-\beta \hbar \times n^{2}} \cosh (\beta \hbar g \sqrt{n+1})}{\sum_{n=0}^{\infty} e^{-\beta \hbar \omega n-\beta \hbar \times n^{2}} \cosh (\beta \hbar g \sqrt{n+1})} .
$$

Finally, introducing Eqs. (37) and (38) into the definition (8) and replacing $\rho_{n}^{* *}$ by $\rho_{n}$, we arrive at the Mandel parameter's expression

$$
\mathcal{Q}_{\mathcal{J C K}}=\frac{\sum_{n=0}^{\infty} n^{2} \rho_{n}^{* *}-\left(\sum_{n=0}^{\infty} n \rho_{n}^{* *}\right)^{2}}{\sum_{n=0}^{\infty} n \rho_{n}^{* *}}-1 .
$$

In Fig. 6 we show the Mandel parameter $\mathcal{Q}_{\mathcal{J C K}}$ in terms of dimensionless temperature $t$ for several values of $\chi / \omega$. The coupling constant $g / \omega$ is fixed at 1.5. The case $\chi / \omega=0$ corresponds to the pure Jaynes-Cummings instance illustrated in Sec. V. In these curves we clearly see that, at some finite temperatures, the Mandel parameter is negative for appropriate $\chi / \omega$ values and thus nonclassical light ensues.

\section{A. Illustration for $T \sim 1 \mathrm{~K}$}

Consider now temperatures low enough that phonon-related effects are not important. If $g / \omega=1.5$, and $\chi / \omega=0$, one has $\mathcal{Q}_{\mathcal{J C K}}<0$ for approximately $0.05<t<0.20$.

If $\chi / \omega=0.1, \mathcal{Q}_{\mathcal{J C K}}<0$ in the interval $0.06<t<0.54$. Thus, for instance, for a frequency $f=1 \times 10^{11} \mathrm{~Hz}$ (wavelength $\lambda=3 \mathrm{~mm})$, and using $T=\left(\hbar \omega / k_{B}\right) t$ one has $\mathcal{Q}_{J C K}<$ 0 in the interval $0.24{ }^{\circ} \mathrm{K}<T<0.96{ }^{\circ} \mathrm{K}$ in the first case, and $0.29{ }^{\circ} \mathrm{K}<T<2.59{ }^{\circ} \mathrm{K}$ in the second one.

\section{CONCLUSION}

In this work we attempted to determine the conditions under which, at finite temperatures, nonclassical radiation may emerge. We deal with:

(i) a humble harmonic oscillator Hamiltonian to which we add a Kerr-ordering one,

(ii) a Jaynes-Cummings Hamiltonian, plus

(iii) adding a Kerr-like term to the JC one.

We were able to ascertain that nonclassical light is here the result of several factors:

(i) molecular alignment, or order, as represented by the Kerr effect,

(ii) finite temperature (i.e., disorder), since it is clear that at $T=0$ we do not reach our sub-Poissonian goal.

(iii) inclusion in the theoretical discussion of fluctuations of order $\hbar^{2}$ and higher, since $\hbar$ effects alone are not sufficient.

Surprisingly enough, some amount of disorder, as represented by a finite temperature, becomes indispensable so as to obtain nonclassical light in the cases here treated. This is the main result of this Brief Report. Nonclassical light arises as the joint collaboration of both quantum plus thermal fluctuations.

\section{ACKNOWLEDGMENTS}

F.P. would like to thank partial financial support by FONDECYT, Grant No. 1110827. This work was also partially supported by the project PIP1177 of CONICET (Argentina), and the projects FIS2008-00781/FIS (MICINN)-FEDER (EU) (Spain, EU).
[1] Daniel W. C. Brooks, T. Botter, S. Schreppler, T. P. Purdy, N. Brahms, and D. M. Stamper-Kurn, Nature (London) 488, 476 (2012).

[2] Quantum Interferometry, edited by F. De Martini et al. (VCH, Weinheim, 1996).

[3] N. Gisin, G. Ribordy, W. Tittel, and H. Zbinden, Rev. Mod. Phys. 74, 145 (2002).

[4] E. Knill, R. Laflamme, and G. J. Milburn, Nature (London) 409, 46 (2001).

[5] P. Zoller et al., Eur. Phys. J. D 36, 203 (2005).

[6] E. C. G. Sudarshan, Phys. Rev. Lett. 10, 277 (1963).
[7] H. J. Kimble, M. Dagenais, and L. Mandel, Phys. Rev. Lett. 39, 691 (1977).

[8] R. Short and L. Mandel, Phys. Rev. Lett. 51, 384 (1983).

[9] G. S. Agarwal and K. Tara, Phys. Rev. A 43, 492 (1991).

[10] W. Schleich and J. A. Wheeler, Nature (London) 326, 574 (1987).

[11] S. Schiller, G. Breitenbach, S. F. Pereira, T. Müller, and J. Mlynek, Phys. Rev. Lett. 77, 2933 (1996).

[12] L. Mandel, Opt. Lett. 4, 205 (1979).

[13] L. Mandel and E. Wolf, Optical coherence and quantum optics (Cambridge University Press, Cambridge, 1995). 
[14] J. Bajer and A. Miranowicz, J. Opt. B 2, L10 (2000).

[15] A. D. Wilson-Gordon, V. Bužek, and P. L. Knight, Phys. Rev. A 44, 7647 (1991).

[16] H. Haus, Waves and Fields Optoelectronics (Prentice Hall, New York, 1984).

[17] M. Melnichuk and L. T. Wood, Phys. Rev. A 82, 013821 (2010).

[18] F. Pennini and A. Plastino, Eur. Phys. J. D. 61, 241 (2011).
[19] F. Pennini, A. Plastino, and G. L. Ferri, Entropy 14, 2081 (2012).

[20] E. T. Jaynes and F. W. Cummings, Proc. IEEE 51, 89 (1963).

[21] H. Paul, Ann. Phys. (Leipzig) 11, 411 (1963).

[22] F. Grusdt (unpublished), available online at http://www. physik.unikl.de/fileadmin/widera/Hauptseminar_WS10_11/ CavityQED.pdf. 